which implies that, $y=T(y)$. This is a contradiction.

Hence, if $\mathrm{y} \neq \mathrm{T}(\mathrm{y})$, then

$$
\inf [G(x, y, y)+G(x, T(x), T(x)): x \in X]>0 .
$$

If $T$ satisfies condition (3.11), then using the same methods as above one can prove that

$$
\inf [G(x, x, y)+G(x, x, T(x)): x \in X]>0 .
$$

So, using Corollary 1, we have the desired result.

The following Corollary is a generalization of the result [[1], Theorem 2.1].

Corollary 3. Let $(\mathrm{X}, \mathrm{G})$ be a complete $\mathrm{G}-$ metric space, and let $\mathrm{T}: \mathrm{X} \rightarrow \mathrm{X}$ be a mapping satisfying one of the following conditions:

$$
\begin{aligned}
& G(T(x), T(y), T(z)) \leq k \max \left\{\begin{array}{l}
G(x, y, z), G(x, T(x), T(x)), \\
G(y, T(y), T(y)), G(z, T(z), T(z)), \\
\frac{G(x, T(y), T(y))+G(z, T(x), T(x))}{2}, \\
\frac{G(x, T(y), T(y))+G(y, T(x), T(x))}{2}, \\
\frac{G(y, T(z), T(z))+G(z, T(y), T(y))}{2}, \\
\frac{G(x, T(z), T(z))+G(z, T(x), T(x))}{2}
\end{array}\right\} \\
& \text { or } \\
& G(T(x), T(y), T(z)) \leq k \max \left\{\begin{array}{l}
G(x, y, z), G(x, x, T(x)), \\
G(y, y, T(y)), G(z, z, T(z)), \\
\frac{G(x, x, T(y))+G(z, z, T(x))}{2}, \\
\frac{G(x, x, T(y))+G(y, y, T(x))}{2}, \\
\frac{G(y, y, T(z))+G(z, z, T(y))}{2}, \\
\frac{G(x, x, T(z))+G(z, z, T(x))}{2}
\end{array}\right\}
\end{aligned}
$$


for all $\mathrm{x}, \mathrm{y}, \mathrm{z} \in \mathrm{X}$, where $\mathrm{O} \leq \mathrm{k}<1$. Then $\mathrm{T}$ has a unique fixed point (say $\mathrm{u}$ ) in $\mathrm{X}$ and $\mathrm{T}$ is $\mathrm{G}$-continuous at $\mathrm{u}$.

Proof. Suppose that $T$ satisfies condition (3.12) for all $x, y, z \in X$. Then replacing $y$ and $z$ by $T(x)$, we obtain from (3.12) and using $\left(\mathrm{G}_{5}\right)$ that

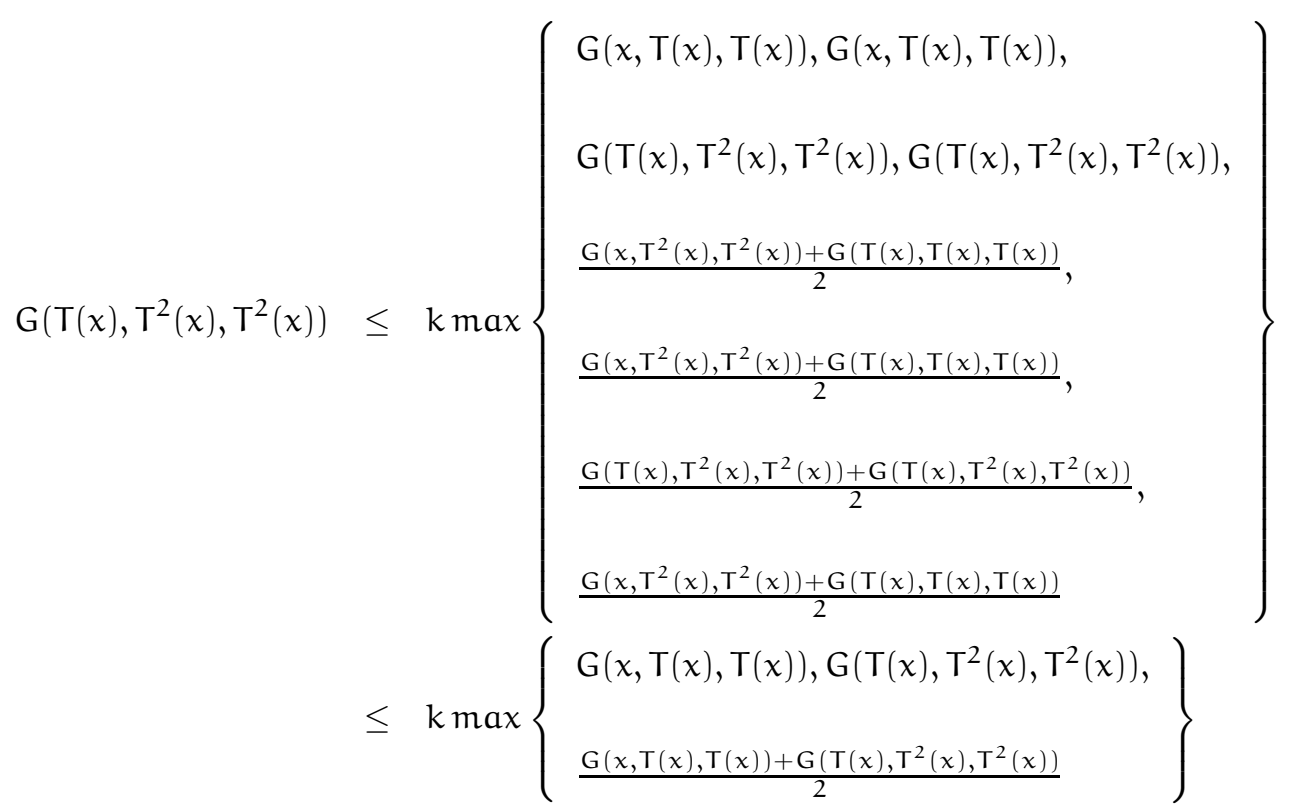

Without loss of generality we may assume that $T(x) \neq T^{2}(x)$. For, otherwise, $T$ has a fixed point. So, (3.14) leads to the following cases,

(1) $G\left(T(x), T^{2}(x), T^{2}(x)\right) \leq k \frac{G(x, T(x), T(x))+G\left(T(x), T^{2}(x), T^{2}(x)\right)}{2}$,

(2) $G\left(T(x), T^{2}(x), T^{2}(x)\right) \leq k G(x, T(x), T(x))$.

In the first case, we have

$$
G\left(T(x), T^{2}(x), T^{2}(x)\right) \leq \frac{k}{2-k} G(x, T(x), T(x)) .
$$

Put $r=\frac{k}{2-k}$. Then $0 \leq r<1$.

Thus, in each case we must have

$$
G\left(T(x), T^{2}(x), T^{2}(x)\right) \leq r G(x, T(x), T(x))
$$

for every $x \in X$, where $0 \leq r<1$.

Assume that there exists $y \in X$ with $y \neq T(y)$ and

$$
\inf [G(x, y, y)+G(x, T(x), T(x)): x \in X]=0 .
$$


Proceeding exactly the same way as in the proof of Corollary 2, there exists a sequence $\left(x_{n}\right)$ in $X$ such that $\left(x_{n}\right)$ is G-convergent to $y$ and $\left(T\left(x_{n}\right)\right)$ is G-convergent to $y$.

Now applying (3.12), we have

$$
G\left(T\left(x_{n}\right), T(y), T(y)\right) \leq k \max \left\{\begin{array}{c}
G\left(x_{n}, y, y\right), G\left(x_{n}, T\left(x_{n}\right), T\left(x_{n}\right)\right), \\
G(y, T(y), T(y)), G(y, T(y), T(y)), \\
\frac{G\left(x_{n}, T(y), T(y)\right)+G\left(y, T\left(x_{n}\right), T\left(x_{n}\right)\right)}{2}, \\
\frac{G\left(x_{n}, T(y), T(y)\right)+G\left(y, T\left(x_{n}\right), T\left(x_{n}\right)\right)}{2}, \\
\frac{G(y, T(y), T(y))+G(y, T(y), T(y))}{2}, \\
\frac{G\left(x_{n}, T(y), T(y)\right)+G\left(y, T\left(x_{n}\right), T\left(x_{n}\right)\right)}{2}
\end{array}\right\} .
$$

Taking the limit as $\mathrm{n} \rightarrow \infty$, and using the fact that the function $\mathrm{G}$ is continuous on its variables, we obtain

$$
G(y, T(y), T(y)) \leq k G(y, T(y), T(y))
$$

which is a contradiction.

Hence, if $y \neq T(y)$, then

$$
\inf [G(x, y, y)+G(x, T(x), T(x)): x \in X]>0 .
$$

Now Corollary 1 applies to obtain a fixed point (say u) of $\mathrm{T}$.

The proof using (3.13) is similar. Uniqueness of $u$ and $\mathrm{G}$-continuity of $\mathrm{T}$ at $\boldsymbol{u}$ may be verified in the usual way by using any one of condition (3.12) and condition (3.13) that $\mathrm{T}$ satisfies.

Remark 1. We see that special cases of Corollary 3 are Theorem 2.1 of [1], Theorem 2.1 of [1] and Theorems 2.1, and 2.4 of [10].

The following Corollary is the result [[1, Theorem 2.2].

Corollary 4. Let $(\mathrm{X}, \mathrm{G})$ be a complete $\mathrm{G}$-metric space, and let $\mathrm{T}: \mathrm{X} \rightarrow \mathrm{X}$ be a mapping satisfying one of the following conditions:

$$
G(T(x), T(y), T(z)) \leq k \max \left\{\begin{array}{l}
G(x, y, z), G(x, T(x), T(x)), \\
G(y, T(y), T(y)), G(x, T(y), T(y)), \\
G(y, T(x), T(x)), G(z, T(z), T(z))
\end{array}\right\}
$$




$$
G(T(x), T(y), T(z)) \leq k \max \left\{\begin{array}{l}
G(x, y, z), G(x, x, T(x)), \\
G(y, y, T(y)), G(x, x, T(y)), \\
G(y, y, T(x)), G(z, z, T(z))
\end{array}\right\}
$$

for all $\mathrm{x}, \mathrm{y}, \mathrm{z} \in \mathrm{X}$, where $\mathrm{O} \leq \mathrm{k}<1$. Then $\mathrm{T}$ has a unique fixed point (say $\mathrm{u}$ ) in $\mathrm{X}$ and $\mathrm{T}$ is $\mathrm{G}$-continuous at $\mathrm{u}$.

Proof. Suppose that T satisfies condition (3.15) for all $x, y, z \in X$. Then replacing $z$ by $x ; y$ and $x$ by $T(x)$ in (3.15), we have

$$
\begin{aligned}
G\left(T^{2}(x), T^{2}(x), T(x)\right) \leq k \max \left\{\begin{array}{l}
G(T(x), T(x), x), G\left(T(x), T^{2}(x), T^{2}(x)\right), \\
G\left(T(x), T^{2}(x), T^{2}(x)\right), G\left(T(x), T^{2}(x), T^{2}(x)\right), \\
G\left(T(x), T^{2}(x), T^{2}(x)\right), G(x, T(x), T(x))
\end{array}\right\} \\
\leq k \max \left\{G(x, T(x), T(x)), G\left(T(x), T^{2}(x), T^{2}(x)\right)\right\} .
\end{aligned}
$$

Without loss of generality we may assume that $T(x) \neq T^{2}(x)$. For, otherwise, $T$ has a fixed point.

So, it must be the case that,

$$
G\left(T(x), T^{2}(x), T^{2}(x)\right) \leq k G(x, T(x), T(x))
$$

for every $x \in X$, where $0 \leq k<1$.

By the same argument used in the proof of Corollary 3 , we see that if $y \neq T(y)$, then

$$
\inf [G(x, y, y)+G(x, T(x), T(x)): x \in X]>0 .
$$

Now Corollary 1 applies to obtain a fixed point (say u) of T.

The proof using (3.16) is similar. Uniqueness of $u$ and $G$-continuity of $T$ at $u$ are obtained by the same argument used in Corollary 3

The following Corollary is the result [[11], Theorem 2.9].

Corollary 5. Let $(\mathrm{X}, \mathrm{G})$ be a complete $\mathrm{G}$-metric space, and let $\mathrm{T}: \mathrm{X} \rightarrow \mathrm{X}$ be a mapping satisfying one of the following conditions:

$$
G(T(x), T(y), T(y)) \leq a\{G(x, T(y), T(y))+G(y, T(x), T(x))\}
$$




$$
\mathrm{G}(\mathrm{T}(\mathrm{x}), \mathrm{T}(\mathrm{y}), \mathrm{T}(\mathrm{y})) \leq \mathrm{a}\{\mathrm{G}(\mathrm{x}, \mathrm{x}, \mathrm{T}(\mathrm{y}))+\mathrm{G}(\mathrm{y}, \mathrm{y}, \mathrm{T}(\mathrm{x}))\}
$$

for all $\mathrm{x}, \mathrm{y} \in \mathrm{X}$, where $\mathrm{O} \leq \mathrm{a}<\frac{1}{2}$. Then $\mathrm{T}$ has a unique fixed point (say $\mathrm{u}$ ) in $\mathrm{X}$ and $\mathrm{T}$ is $\mathrm{G}$-continuous at $\mathrm{u}$.

Proof. Suppose that $T$ satisfies condition (3.17) for all $x, y \in X$. Then replacing y by $T(x)$ in (3.17), we have

$$
\begin{aligned}
G\left(T(x), T^{2}(x), T^{2}(x)\right) & \leq a\left\{G\left(x, T^{2}(x), T^{2}(x)\right)+G(T(x), T(x), T(x))\right\} \\
& \leq a\left\{G(x, T(x), T(x))+G\left(T(x), T^{2}(x), T^{2}(x)\right)\right\}, \text { by }\left(G_{5}\right) .
\end{aligned}
$$

So, it must be the case that,

$$
G\left(T(x), T^{2}(x), T^{2}(x)\right) \leq \frac{a}{1-a} G(x, T(x), T(x)) .
$$

Put $r=\frac{a}{1-a}$. Then $0 \leq r<1$ since $0 \leq a<\frac{1}{2}$.

Thus,

$$
G\left(T(x), T^{2}(x), T^{2}(x)\right) \leq r G(x, T(x), T(x)) .
$$

for every $x \in X$, where $0 \leq r<1$.

Assume that there exists $y \in X$ with $y \neq T(y)$ and

$$
\inf [G(x, y, y)+G(x, T(x), T(x)): x \in X]=0 .
$$

As in the proof of Corollary 2, there exists a sequence $\left(x_{n}\right)$ in $X$ such that $\left(x_{n}\right)$ is G-convergent to $y$ and $\left(T\left(x_{n}\right)\right)$ is G-convergent to $y$.

Now using (3.17), we have

$$
G\left(T\left(x_{n}\right), T(y), T(y)\right) \leq a\left\{G\left(x_{n}, T(y), T(y)\right)+G\left(y, T\left(x_{n}\right), T\left(x_{n}\right)\right)\right\} .
$$

Taking the limit as $\mathrm{n} \rightarrow \infty$, and using the fact that the function $\mathrm{G}$ is continuous on its variables, we have

$$
\begin{aligned}
G(y, T(y), T(y)) & \leq a\{G(y, T(y), T(y))+G(y, y, y)\} \\
& =a G(y, T(y), T(y)),
\end{aligned}
$$

which is a contradiction.

Hence, if $y \neq T(y)$, then

$$
\inf [G(x, y, y)+G(x, T(x), T(x)): x \in X]>0 .
$$

Now applying Corollary 1 , we obtain a fixed point (say u) of $\mathrm{T}$.

The proof using (3.18) is similar. Uniqueness of $u$ and $G$-continuity of $T$ at $u$ are obtained by the same argument used above.

Received: May 2011. Revised: June 2012. 


\section{References}

[1] R.Chugh, T.Kadian, A.Rani, and B.E.Rhoades, "Property P in G-metric spaces," Fixed Point Theory and Applications, vol. 2010, Article ID 401684, 12 pages, 2010.

[2] B.C.Dhage, "Generalised metric spaces and mappings with fixed point," Bulletin of the Calcutta Mathematical Society, vol.84, no. 4, pp. 329-336, 1992.

[3] B.C.Dhage, "Generalised metric spaces and topological structure- I," Analele Stiintifice ale Universitătii "Al.I.Cuza" din Iasi. Serie Nouă. Matematică, vol.46, no. 1, pp. 3-24, 2000.

[4] S.Gähler, "2-metrische Räume und ihre topologische Struktur," Mathematische Nachrichten, vol.26, pp. 115-148, 1963.

[5] S.Gähler, "Zur geometric 2-metrische räume," Revue Roumaine de Mathématiques Pures et Appliquées, vol.40, pp. 664-669, 1966.

[6] K.S.Ha,Y.J.Cho, and A.White, "Strictly convex and strictly 2-convex 2-normed spaces," Mathematica Japonica, vol.33, no. 3, pp. 375-384, 1988.

[7] Osamu Kada, Tomonari Suzuki and Wataru Takahashi, "Nonconvex minimization theorems and fixed point theorems in complete metric spaces," Math. Japonica, vol.44, no. 2, pp. 381-391, 1996,.

[8] Sushanta Kumar Mohanta, "Property P of Ćirić operators in G-metric spaces," International J. of Math. Sci. and Engg. Appls., vol. 5, no. II, pp. 353-367, 2011.

[9] Z.Mustafa and B.Sims, "A new approach to generalized metric spaces," Journal of Nonlinear and convex Analysis, vol. 7, no. 2, pp. 289-297, 2006.

[10] Z.Mustafa and B.Sims, "Fixed point theorems for contractive mappings in complete G-metric spaces," Fixed Point Theory and Applications, vol. 2009, Article ID 917175, 10 pages, 2009.

[11] Z.Mustafa, H.Obiedat, and F. Awawdeh, "Some fixed point theorem for mapping on complete G-metric spaces," Fixed Point Theory and Applications, vol. 2008, Article ID 189870, 12 pages, 2008.

[12] Z.Mustafa, W.Shatanawi, and M.Bataineh, "Existence of fixed point results in G-metric spaces," International Journal of Mathematics and Mathematical Sciences, vol. 2009, Article ID 283028, 10 pages, 2009.

[13] Z.Mustafa and B.Sims, "Some remarks concerning D-metric spaces," in Proceedings of the International Conference on Fixed Point Theory and Applications, pp. 189-198, Valencia, Spain, July 2004.

[14] Z.Mustafa, A new structure for generalized metric spaces-with applications to fixed point theory, Ph.D. thesis, The University of Newcastle, Callaghan, Australia, 2005. 
[15] Z.Mustafa and H. Obiedat, "A fixed points theorem of Reich in G-metric spaces," Cubo A Mathematics Journal, vol. 12, no. 01, pp. 83-93, 2010.

[16] Z.Mustafa, F. Awawdeh and W.Shatanawi, "Fixed point theorem for expansive mappings in G-metric spaces," Int. J. Contemp. Math. Sciences, vol. 5, no. 50, pp. 2463-2472, 2010.

[17] S.V.R.Naidu, K.P.R.Rao, and N.Srinivasa Rao, "On the concept of balls in a D-metric space," International Journal of Mathematics and Mathematical Sciences, no. 1, pp. 133-141, 2005.

[18] W.Shatanawi,"Fixed point theory for contractive mappings satisfying $\phi$-maps in G-metric spaces," Fixed Point Theory and Applications, vol. 2010, Article ID 181650, 9 pages, 2010. 

CUBO A Mathematical Journal

Vol.14, No 03 , (103-113). October 2012

\title{
An Elementary Study of a Class of Dynamic Systems with Two Time Delays
}

\author{
Akio Matsumoto 1 \\ Department of Economics, \\ Chuo University, \\ 742-1, Higashi-Nakano, Hachioji, Tokyo, \\ 192-0393, Japan \\ email: akiom@tamacc.chuo-u.ac.jp
}

\author{
FERENC SzIDAROVSZKY \\ Department of Systems and Industrial \\ Engineering, \\ University of Arizona, \\ Tucson, 85721-0020, USA. \\ email: szidar@sie.arizona.edu
}

\begin{abstract}
An elementary analysis is developed to determine the stability region of a certain class of ordinary differential equations with two delays. Our analysis is based on determining stability switches first where an eigenvalue is pure complex, and then checking the conditions for stability loss or stability gain. In the case of both stability losses and stability gains Hopf bifurcation occurs giving the possibility of the birth of limit cycles.
\end{abstract}

\section{RESUMEN}

Se realiza un análisis básico para determinar la estabilidad de la región de una cierta clase de ecuaciones diferenciales ordinaras con dos retrasos. Nuestro análisis se basa en la determinación de switches de estabilidad, en primer lugar cuando un autovalor es complejo puro, y luego revisando las condiciones para la pérdida o ganancia de estabilidad. En el caso de ambas pérdidas de estabilidad y ganancias de estabilidad, se obtiene la bifurcación de Hopf dando la posibilidad del nacimiento de ciclos límites.

Keywords and Phrases: dynamic systems, time delays, stabiliy analysis.

2010 AMS Mathematics Subject Classification: 34K20, 37C75

1 The authors highly appreciate financial supports from the Japan Society for the Promotion of Science (Grantin-Aid for Scientific Research (C) 24530202) and Chuo University (Grant for Special Research and Joint Research Grant 0981). 


\section{Introduction}

Dynamic models with time delays have many applications in many fields of quantitative sciences (see for example, Cushing (1977) and Invernizzi and Medio (1991)). The case of a single delay is well established in the literature (Hayes (1950) and Burger (1956)), however the presence of multiple delays makes analysis much more complicated. Sufficient and necessary conditions were derived for several classes of models giving a complete description of the stability region (Hale (1979), Hale and Huang (1993) and Piotrowska (2007)).

In this paper a special class of dynamic systems is considered which are governed by delay differential equations with two delays. It is well known (Hayes (1950) and Cooke and Grossman (1982)) that stability can be lost or gained on a curve of stability switches, where an eigenvalue is pure complex. We will therefore determine these curves and then by bifurcation analysis characterize those segments where stability is gained or lost. In this way the stability region can be completely described.

This paper is the continuation of our previous work (Matsumoto and Szidarovszky (2011)) where an elementary analysis was presented with a single delay.

The paper is organized in the following way. Section 2 determines the curves where stability switches are possible and characterizes those segments where stability is lost or gained in the nonsymmetric cases. Section 3 discusses the symmetric case and Section 4 concludes the paper.

\section{Stability Switches and Stability Region}

We will examine the asymptotical stability of the delay differential equation

$$
\dot{x}(t)+A x\left(t-\tau_{1}\right)+B x\left(t-\tau_{2}\right)=0
$$

where $A$ and $B$ are positive constants. The characteristic equation can be obtained by looking for the solution in the exponential form $\alpha e^{\lambda t}$. By substitution,

$$
\alpha \lambda e^{\lambda t}+A \alpha e^{\lambda\left(t-\tau_{1}\right)}+B \alpha e^{\lambda\left(t-\tau_{2}\right)}=0
$$

or

$$
\lambda+\mathrm{A} e^{-\lambda \tau_{1}}+\mathrm{B} e^{-\lambda \tau_{2}}=0 .
$$

Introduce the new variables

$$
\begin{gathered}
\omega=\frac{A}{A+B}, 1-\omega=\frac{B}{A+B}, \bar{\lambda}=\frac{\lambda}{A+B} \\
\gamma_{1}=\tau_{1}(A+B) \text { and } \gamma_{2}=\tau_{2}(A+B)
\end{gathered}
$$

to reduce equation (2.2) to the following:

$$
\bar{\lambda}+\omega e^{-\bar{\lambda} \gamma_{1}}+(1-\omega) e^{-\bar{\lambda} \gamma_{2}}=0 .
$$


Because of symmetry we can assume that $\omega \geq 1 / 2$.In order to find the stability region in the $\left(\gamma_{1}, \gamma_{2}\right)$ plane we will first characterize the cases when an eigenvalue is pure complex, that is, when $\bar{\lambda}=\mathfrak{i} v$. We can assume that $v>0$, since if $\bar{\lambda}$ is an eigenvalue, its complex conjugate is also an eigenvalue. Substituting $\bar{\lambda}=\mathfrak{i v}$ into equation (2.3) we have

$$
\mathfrak{w}+\omega e^{-i v \gamma_{1}}+(1-\omega) e^{-i v \gamma_{2}}=0 .
$$

In the special case of $\gamma_{1}=0$, the equation becomes

$$
\mathfrak{w}+\omega+(1-\omega) e^{-i v \gamma_{2}}=0 .
$$

The real and imaginary parts imply that

$$
\begin{aligned}
& \omega+(1-\omega) \cos \left(v \gamma_{2}\right)=0 \\
& v-(1-\omega) \sin \left(v \gamma_{2}\right)=0 .
\end{aligned}
$$

We can assume first $\omega>1 / 2$, so from the first equation

$$
\cos \left(v \gamma_{2}\right)=-\frac{\omega}{1-\omega}<-1
$$

so no stability switch is possible. If $\omega=1 / 2$, then

$$
\cos \left(v \gamma_{2}\right)=-1
$$

implying that $\sin \left(v \gamma_{2}\right)=0$ and so $v=0$ showing that there is no pure complex root. Hence for $\gamma_{1}=0$ the system is asymptotically stable with all $\gamma_{2} \geq 0$.

Assume now that $\gamma_{1}>0, \gamma_{2} \geq 0$. The real and imaginary parts give two equations:

$$
\omega \cos \left(v \gamma_{1}\right)+(1-\omega) \cos \left(v \gamma_{2}\right)=0
$$

and

$$
v-\omega \sin \left(v \gamma_{1}\right)-(1-\omega) \sin \left(v \gamma_{2}\right)=0 .
$$

We consider the case of $\omega>1 / 2$ first and the symmetric case of $\omega=1 / 2$ will be discussed later. Introduce the variables

$$
x=\sin \left(v \gamma_{1}\right) \text { and } y=\sin \left(v \gamma_{2}\right)
$$

then (2.4) implies that

$$
\omega^{2}\left(1-x^{2}\right)=(1-\omega)^{2}\left(1-y^{2}\right)
$$

or

$$
-\omega^{2} x^{2}+(1-\omega)^{2} y^{2}=1-2 \omega
$$

From (2.5),

$$
v-\omega x-(1-\omega) y=0
$$


implying that

$$
y=\frac{v-\omega x}{1-\omega}
$$

Combining (2.6) and (2.7) yields

$$
-\omega^{2} x^{2}+(1-\omega)^{2}\left(\frac{v-\omega x}{1-\omega}\right)^{2}=1-2 \omega
$$

from which we can conclude that

$$
x=\frac{v^{2}+2 w-1}{2 v w}
$$

and then from (2.7),

$$
y=\frac{v^{2}-2 w+1}{2 v(1-w)}
$$

Equations (2.8) and (2.9) provide a parameterized curve in the $\left(\gamma_{1}, \gamma_{2}\right)$ plane:

$$
\sin \left(v \gamma_{1}\right)=\frac{v^{2}+2 \omega-1}{2 v \omega} \text { and } \sin \left(v \gamma_{2}\right)=\frac{v^{2}-2 \omega+1}{2 v(1-\omega)}
$$

In order to guarantee feasibility we have to satisfy

$$
-1 \leq \frac{v^{2}+2 w-1}{2 v w} \leq 1
$$

and

$$
-1 \leq \frac{v^{2}-2 w+1}{2 v(1-w)} \leq 1
$$

Simple calculation shows that with $\omega>1 / 2$ these relations hold if and only if

$$
2 \omega-1 \leq v \leq 1
$$

From (2.10) we have four cases for $\gamma_{1}$ and $\gamma_{2}$, since

$$
\gamma_{1}=\frac{1}{v}\left\{\sin ^{-1}\left(\frac{v^{2}+2 \omega-1}{2 v \omega}\right)+2 k \pi\right\}
$$

or

$$
\gamma_{1}=\frac{1}{v}\left\{\pi-\sin ^{-1}\left(\frac{v^{2}+2 \omega-1}{2 v \omega}\right)+2 k \pi\right\} \quad(k=0,1,2, \ldots)
$$

and similarly

$$
\gamma_{2}=\frac{1}{v}\left\{\sin ^{-1}\left(\frac{v^{2}-2 \omega+1}{2 v(1-\omega)}\right)+2 n \pi\right\}
$$

or

$$
\gamma_{2}=\frac{1}{v}\left\{\pi-\sin ^{-1}\left(\frac{v^{2}-2 \omega+1}{2 v(1-\omega)}\right)+2 n \pi\right\} \quad(n=0,1,2, \ldots) .
$$


However from (2.4) we can see that $\cos \left(v \gamma_{1}\right)$ and $\cos \left(v \gamma_{2}\right)$ must have different signs, so we have only two possibilities:

$$
\mathrm{L}_{1}(\mathrm{k}, \mathrm{n}):\left\{\begin{array}{l}
\gamma_{1}=\frac{1}{v}\left\{\sin ^{-1}\left(\frac{v^{2}+2 \omega-1}{2 v \omega}\right)+2 k \pi\right\} \\
\gamma_{2}=\frac{1}{v}\left\{\pi-\sin ^{-1}\left(\frac{v^{2}-2 \omega+1}{2 v(1-\omega)}\right)+2 n \pi\right\}
\end{array}\right.
$$

and

$$
\mathrm{L}_{2}(\mathrm{k}, \mathrm{n}):\left\{\begin{array}{l}
\gamma_{1}=\frac{1}{v}\left\{\pi-\sin ^{-1}\left(\frac{v^{2}+2 \omega-1}{2 v \omega}\right)+2 \mathrm{k} \pi\right\} \\
\gamma_{2}=\frac{1}{v}\left\{\sin ^{-1}\left(\frac{v^{2}-2 \omega+1}{2 v(1-\omega)}\right)+2 \mathrm{n} \pi\right\}
\end{array}\right.
$$

For each $v \in[2 \omega-1,1]$ these equations determine the values of $\gamma_{1}$ and $\gamma_{2}$. At the initial point $v=2 \omega-1$, we have

$$
\frac{v^{2}+2 w-1}{2 v w}=1 \text { and } \frac{v^{2}-2 w+1}{2 v(1-w)}=-1
$$

and if $v=1$, then

$$
\frac{v^{2}+2 w-1}{2 v \omega}=1 \text { and } \frac{v^{2}-2 \omega+1}{2 v(1-\omega)}=1 .
$$

Therefore the starting point and end point of $L_{1}(k, n)$ are given as

$$
\gamma_{1}^{s}=\frac{1}{2 \omega-1}\left(\frac{\pi}{2}+2 k \pi\right), \gamma_{2}^{s}=\frac{1}{2 \omega-1}\left(\frac{3 \pi}{2}+2 n \pi\right)
$$

and

$$
\gamma_{1}^{e}=\frac{\pi}{2}+2 k \pi \text { and } \gamma_{2}^{e}=\frac{\pi}{2}+2 n \pi
$$

Similarly, the starting and end points of $L_{2}(k, n)$ are as follows:

$$
\gamma_{1}^{S}=\frac{1}{2 \omega-1}\left(\frac{\pi}{2}+2 k \pi\right), \gamma_{2}^{S}=\frac{1}{2 \omega-1}\left(-\frac{\pi}{2}+2 n \pi\right)
$$

and

$$
\gamma_{1}^{\mathrm{E}}=\frac{\pi}{2}+2 \mathrm{k} \pi \text { and } \gamma_{2}^{\mathrm{E}}=\frac{\pi}{2}+2 n \pi .
$$

Figure 1 illustrates the loci $L_{1}(k, n)$ and $L_{2}(k, n)$ of the corresponding points $\left(\gamma_{1}, \gamma_{2}\right)$, when $v$ increases from $2 \omega-1$ to unity. The parameter value $\omega=0.8$ is selected. The red curves show $\mathrm{L}_{1}(0, n)$ and the blue curves show $\mathrm{L}_{2}(0, n)$ with $\mathrm{n}=0,1,2, \ldots$. Notice that $\gamma_{2}^{S}$ is infeasible at $\mathrm{n}=0$ and only the segment of $L_{2}(0,0)$ between $v=\sqrt{2 \omega-1}$ and $v=1$ is feasible. With fixed value of $k, L_{1}(k, n)$ and $L_{2}(k, n)$ have the same end point, however the starting point of $L_{1}(k, n)$ is the same as that of $L_{2}(k, n+1)$. Therefore the segments $L_{1}(k, n)$ and $L_{2}(k, n)$ with fixed $k$ form a continuous curve with $n=0,1,2, \ldots$ 


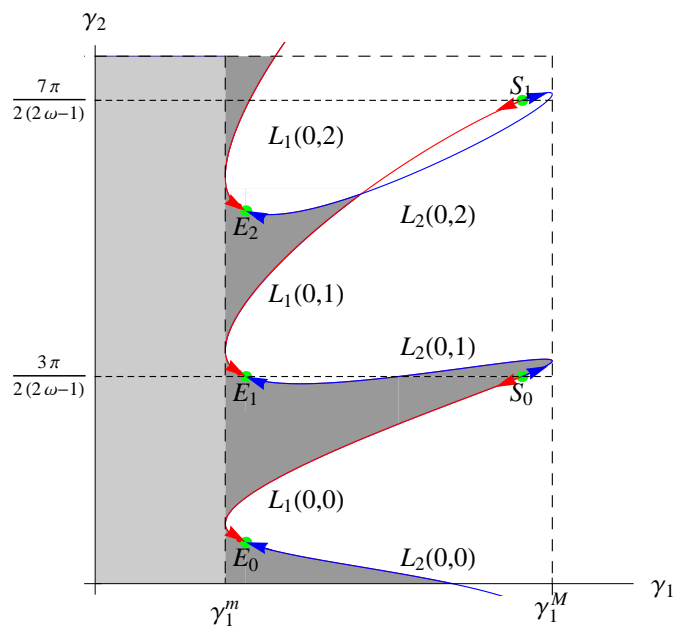

Figure 1. Partition curve in the $\left(\gamma_{1}, \gamma_{2}\right)$ plane with fixing $k=0$.

Consider first the segment $L_{1}(k, n)$. Since $\left(v^{2}-2 w+1\right) /(2 v(1-w))$ is strictly increasing in $v, \gamma_{2}$ is strictly decreasing in $v$. By differentiation and substitution of equation (2.4), we have

$$
\begin{aligned}
\left.\frac{\partial \gamma_{1}}{\partial v}\right|_{\mathrm{L}_{1}} & =-\frac{1}{v^{2}}\left(\sin ^{-1}\left(\frac{v^{2}+2 \omega-1}{2 v \omega}\right)+2 k \pi\right)+\frac{1}{v \sqrt{1-\left(\frac{v^{2}+2 \omega-1}{2 v \omega}\right)^{2}}} \frac{2 v(2 v \omega)-\left(v^{2}+2 \omega-1\right) 2 \omega}{2^{2} v^{2} \omega^{2}} \\
& =-\frac{1}{v^{2}} v \gamma_{1}+\frac{1}{v \cos \left(v \gamma_{1}\right)} \frac{v^{2}-2 \omega+1}{2 v^{2} \omega} \\
& =-\frac{1}{v^{2}}\left(v \gamma_{1}+\tan \left(v \gamma_{2}\right)\right) .
\end{aligned}
$$

Consider next segment $L_{2}(k, n)$, similarly to (2.15) we can shown that

$$
\left.\frac{\partial \gamma_{1}}{\partial v}\right|_{L_{2}}=-\frac{1}{v^{2}}\left(v \gamma_{1}+\tan \left(v \gamma_{2}\right)\right)
$$

which is the same as in $L_{1}(k, n)$, since from (2.14), $\cos \left(v \gamma_{1}\right)<0$. Similarly

$$
\left.\frac{\partial \gamma_{2}}{\partial v}\right|_{L_{2}}=-\frac{1}{v^{2}}\left(v \gamma_{2}+\tan \left(v \gamma_{1}\right)\right)
$$

where we used again equation (2.4).

In order to visualize the curves $L_{1}(k, n)$ and $L_{2}(k, n)$, we change the coordinates $\left(\gamma_{1}, \gamma_{2}\right)$ to $\left(v \gamma_{1}, v \gamma_{2}\right)$ to get the transformed segments:

$$
\ell_{1}(k, n):\left\{\begin{array}{l}
v \gamma_{1}=\sin ^{-1}\left(\frac{v^{2}+2 \omega-1}{2 v \omega}\right)+2 k \pi \\
v \gamma_{2}=\pi-\sin ^{-1}\left(\frac{v^{2}-2 \omega+1}{2 v(1-\omega)}\right)+2 n \pi
\end{array}\right.
$$


and

$$
\ell_{2}(k, n):\left\{\begin{array}{l}
v \gamma_{1}=\pi-\sin ^{-1}\left(\frac{v^{2}+2 \omega-1}{2 v \omega}\right)+2 k \pi \\
v \gamma_{2}=\sin ^{-1}\left(\frac{v^{2}-2 \omega+1}{2 v(1-\omega)}\right)+2 n \pi
\end{array}\right.
$$

They also form a continuous curve with each fixed value of $k$, and they are periodic in both directions $v \gamma_{1}$ and $v \gamma_{2}$. Figure 2 shows them with $k=0$ where the curves $\ell_{1}(0, n)$ are shown in red color while the curves $\ell_{2}(0, n)$ with blue color.

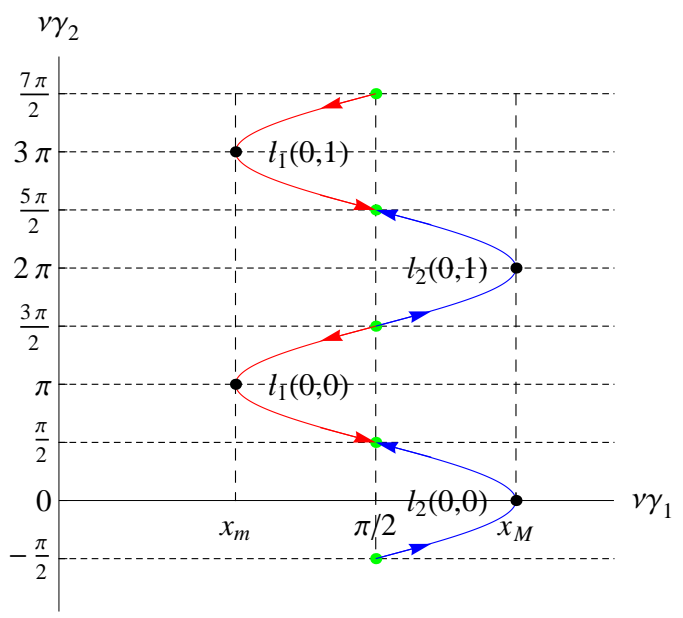

Figure 2. Partition curve in the $\left(v \gamma_{1}, v \gamma_{2}\right)$ plane with fixing $k=0$

We will next examine the directions of the stability switches on the different segments of the curves $L_{1}(k, n)$ and $L_{2}(k, n)$. We fix the value of $\gamma_{2}$ and select $\gamma_{1}$ as the bifurcation parameter, so the eigenvalues are functions of $\gamma_{1}: \bar{\lambda}=\lambda\left(\gamma_{1}\right)$. By differentiating the characteristic equation (2.3) implicitly with respect to $\gamma_{1}$ we have

$$
\frac{\mathrm{d} \bar{\lambda}}{\mathrm{d} \gamma_{1}}+\omega e^{-\bar{\lambda} \gamma_{1}}\left(-\frac{\mathrm{d} \bar{\lambda}}{\mathrm{d} \gamma_{1}} \gamma_{1}-\bar{\lambda}\right)+(1-\omega) e^{-\bar{\lambda} \gamma_{2}}\left(-\frac{\mathrm{d} \bar{\lambda}}{\mathrm{d} \gamma_{1}} \gamma_{2}\right)=0
$$

implying that

$$
\frac{d \bar{\lambda}}{d \gamma_{1}}=\frac{\bar{\lambda} \omega e^{-\bar{\lambda} \gamma_{1}}}{1-\omega \gamma_{1} e^{-\bar{\lambda} \gamma_{1}}-(1-\omega) \gamma_{2} e^{-\bar{\lambda} \gamma_{2}}}
$$

From (2.3) we have

$$
(1-\omega) e^{-\bar{\lambda} \gamma_{2}}=-\bar{\lambda}-\omega e^{-\bar{\lambda} \gamma_{1}}
$$

so

$$
\frac{\mathrm{d} \bar{\lambda}}{\mathrm{d} \gamma_{1}}=\frac{\bar{\lambda} \omega e^{-\bar{\lambda} \gamma_{1}}}{1+\bar{\lambda} \gamma_{2}+\omega\left(\gamma_{2}-\gamma_{1}\right) e^{-\bar{\lambda} \gamma_{1}}}
$$


If $\bar{\lambda}=\mathfrak{v}$, then

$$
\frac{\mathrm{d} \bar{\lambda}}{\mathrm{d} \gamma_{1}}=\frac{\mathfrak{i v \omega}\left(\cos \left(v \gamma_{1}\right)-\mathfrak{i} \sin \left(v \gamma_{1}\right)\right)}{1+i v \gamma_{2}+\omega\left(\gamma_{2}-\gamma_{1}\right)\left(\cos \left(v \gamma_{1}\right)-i \sin \left(v \gamma_{1}\right)\right)}
$$

and the real part of this expression has the same sign as

$$
\begin{gathered}
v \omega \sin \left(v \gamma_{1}\right)\left[1+\omega\left(\gamma_{2}-\gamma_{1}\right) \cos \left(v \gamma_{1}\right)\right]+v \omega \cos \left(v \gamma_{1}\right)\left[v \gamma_{2}-\omega\left(\gamma_{2}-\gamma_{1}\right) \sin \left(v \gamma_{1}\right)\right] \\
=v \omega\left[\sin \left(v \gamma_{1}\right)+v \gamma_{2} \cos \left(v \gamma_{1}\right)\right]
\end{gathered}
$$

Hence

$$
\operatorname{Re}\left(\frac{\mathrm{d} \bar{\lambda}}{\mathrm{d} \gamma_{1}}\right) \gtreqless 0 \text { if and only if } \sin \left(v \gamma_{1}\right)+v \gamma_{2} \cos \left(v \gamma_{1}\right) \gtreqless 0
$$

Consider first the case of crossing any segment $L_{1}(k, n)$ from the left. Here $v \gamma_{1} \in(0, \pi / 2]$, so both $\sin \left(v \gamma_{1}\right)$ and $\cos \left(v \gamma_{2}\right)$ are positive. Hence stability is lost everywhere on any segment of $L_{1}(k, n)$. Consider the case when crossing the segments of $L_{2}(k, n)$ from the left. Here $v \gamma_{1} \in[\pi / 2, \pi]$, so $\cos \left(v \gamma_{1}\right)<0$. Combining (2.16) and the conditions for the sign of $\operatorname{Re}\left[\mathrm{d} \bar{\lambda} / \mathrm{d} \gamma_{1}\right]$, we have that

$$
\operatorname{Re}\left(\frac{\mathrm{d} \bar{\lambda}}{\mathrm{d} \gamma_{1}}\right) \gtreqless 0 \text { if and only if } \frac{\partial \gamma_{2}}{\partial v} \gtreqless 0 \text {. }
$$

That is, stability is lost when $\gamma_{2}$ increases in $v$ and stability is gained when $\gamma_{2}$ decreases in $v$. We can also show that at any intercept with $L_{1}(k, n)$ or $L_{2}(k, n)$ the complex root is single. Otherwise $\lambda=\mathfrak{i} v$ would satisfy both equations

$$
\lambda+\omega e^{-\lambda \gamma_{1}}+(1-\omega) e^{-\lambda \gamma_{2}}=0
$$

and

$$
1-\omega \gamma_{1} e^{-\lambda \gamma_{1}}-(1-\omega) \gamma_{2} e^{-\lambda \gamma_{2}}=0
$$

from which we have

$$
e^{-\lambda \gamma_{1}}=\frac{1+\lambda \gamma_{2}}{\left(\gamma_{1}-\gamma_{2}\right) \omega} \text { and } e^{-\lambda \gamma_{2}}=\frac{-1-\lambda \gamma_{1}}{\left(\gamma_{1}-\gamma_{2}\right)(1-\omega)}
$$

By substituting $\lambda=\mathfrak{i v}$ and comparing the real and imaginary parts yield

$$
\sin \left(v \gamma_{1}\right)+v \gamma_{2} \cos \left(v \gamma_{1}\right)=\sin \left(v \gamma_{2}\right)+v \gamma_{1} \cos \left(v \gamma_{2}\right)=0
$$

Therefore this intercept is at an extremum in $v$ of a segment $L_{1}(k, n)$ and also at an extremum of a segment $L_{2}(\bar{k}, \bar{n})$ which is impossible.

For each $\gamma_{2}>0$, define

$$
m\left(\gamma_{2}\right)=\min _{\gamma_{1}}\left\{\left(\gamma_{1}, \gamma_{2}\right) \in L_{1}(k, n) \cup L_{2}(k, n), k, n \geq 0\right\}
$$

At $\gamma_{1}=0$ the system is asymptotically stable with all $\gamma_{2}>0$. With fixed value of $\gamma_{2}$ by increasing the value of $\gamma_{1}$ the first intercept with $m\left(\gamma_{2}\right)$ should be a stability loss, since there is no stability switch before. Then by increasing the value of $\gamma_{1}$ further, the next intercept is either a stability 
gain or a stability loss. In the first case the equilibrium becomes asymptotically stable. In the second case the equilibrium remains unstable, which will not change even if the next intercept is an stability gain, since the real part of only one eigenvalue becomes negative.

Consider next a point $\left(\gamma_{1}^{*}, \gamma_{2}^{*}\right)$ with $\gamma_{1}^{*}, \gamma_{2}^{*}>0$ which is not located on any curve $L_{1}(k, n)$ or $\mathrm{L}_{2}(k, n)$, and consider the horizontal line $\gamma_{2}=\gamma_{2}^{*}$ and its segment with $\gamma_{1} \in\left(0, \gamma_{1}^{*}\right)$. If it has no stability switch, then the equilibrium is asymptotically stable. This is the case even if the number of stability losses equals the number of stability gains, otherwise the equilibrium is unstable. The stability region is shown as the shaded region in Figure 1. Notice that this is the same result which was obtained earlier by Hale and Huang (1993) by using different approach.

\section{The Symmetric Case}

Assume next that $\omega=1 / 2$. Then equations (2.4) and (2.5) imply that

$$
\begin{gathered}
\cos \left(v \gamma_{1}\right)+\cos \left(v \gamma_{2}\right)=0 \\
v-\frac{1}{2}\left(\sin \left(v \gamma_{1}\right)+\sin \left(v \gamma_{2}\right)\right)=0
\end{gathered}
$$

and the curves $L_{1}(k, n)$ and $L_{2}(k, n)$ are simplified as follows:

$$
\mathrm{L}_{1}(k, n):\left\{\begin{array}{l}
\gamma_{1}=\frac{1}{v}\left(\sin ^{-1}(v)+2 k \pi\right) \\
\gamma_{2}=\frac{1}{v}\left(\pi-\sin ^{-1}(v)+2 n \pi\right)
\end{array}\right.
$$

and

$$
\mathrm{L}_{2}(k, n):\left\{\begin{array}{l}
\gamma_{1}=\frac{1}{v}\left(\pi-\sin ^{-1}(v)+2 k \pi\right) \\
\gamma_{2}=\frac{1}{v}\left(\sin ^{-1}(v)+2 n \pi\right)
\end{array}\right.
$$

which are shown in Figure 3. The same argument as shown above for the nonsymmetric case can be applied here as well to show that stability region is left of $L_{1}(0,0)$ and below $L_{2}(0,0)$, where the shape of the stability region differs from that of the nonsymmetric case. It is illustrated in Figure 3 by the shaded domain. 


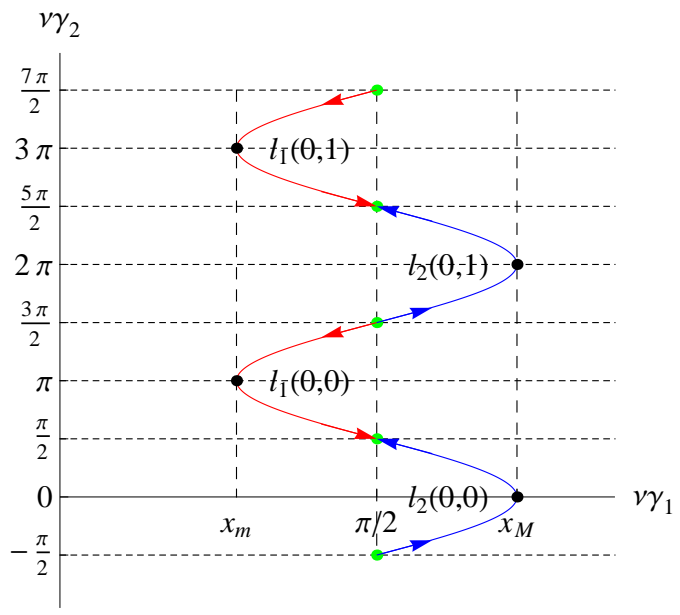

Figure 3. Partition curve in the $\left(\gamma_{1}, \gamma_{2}\right)$ plane with $\omega=\frac{1}{2}$

Notice that at each segment of $\ell_{2}(k, n)$ there are at most two intercepts with the $v \gamma_{2}=$ $-\tan \left(v \gamma_{1}\right)$ curve, so the same holds for $L_{2}(k, n)$. At every other point $\operatorname{Re}\left[d \bar{\lambda} / d \gamma_{1}\right] \neq 0$, so at these points Hopf bifurcation occurs giving the possibility of the birth of limit cycles.

\section{Conclusions}

Ordinary differential equation were examined with two delays. After finding the possible stability switches, their curves were determined. Hopf bifurcation was used to find segments with stability losses and stability gains. The boundary of the stability region are the $\gamma_{2}=0, \gamma_{1}=0$ and a continuous curve consisting of certain portions of the segments $L_{1}(0, n)$ and $L_{2}(0, n)$. All other points on the curves $L_{1}(k, n)$ and $L_{2}(k, n)$ for $k \geq 1$ do not lead to actual stability switches, since the system is already unstable.

\section{Received: July 2011. Revised: June 2012.}

\section{References}

[1] Burger, E. (1956), On the Stability of Certain Economic Systems. Econometrica, 24(4), 488-493.

[2] Cooke, K. L. and Z. Grossman (1982), Discrete Delay, Distributed Delay and Stability Switches. J. of Math. Analysis and Appl., 86, 592-627.

[3] Cushing, J. M. (1977), Integro-Differential Equations and Delay Models in Population Dynamics. Springer-Verlag, Berlin/Heidelberg/New York.

[4] Hayes, N. D. (1950), Roots of the Transcendental Equation Associated with a Certain Difference-Differential Equation. J. of the London Math. Society, 25, 226-232. 
[5] Hale, J. (1979), Nonlinear Oscillations in Equations with Delays. In Nonlinear Oscillations in Biology (K. C. Hoppenstadt, ed.). Lectures in Applied Mathematics, 17, 157-185.

[6] Hale, J. and W. Huang (1993), Global Geometry of the Stable Regions for Two Delay Differential Equations. J. of Math. Analysis and Appl., 178, 344-362.

[7] Invernizzi, S. and A. Medio (1991), On Lags and Chaos in Economic Dynamic Models. Journal of Math. Econ., 20, 521-550.

[8] Matsumoto, A. and F. Szidarovszky (2011), An Elementary Study of a Class of Dynamic Systems with Single Delay. Mimeo: DP 161 (http://www2.chuo-u.ac.jp/keizaiken/discuss.htm), Institute of Economic Research, Chuo University, Tokyo, Japan.

[9] Piotrowska, M. (2007), A Remark on the ODE with Two Discrete Delays. Journal of Math. Analysis and Appl., 329, 664-676. 